\title{
Management of Intraarticular Calcaneal Fractures by Minimally Invasive Sinus Tarsi Approach-Early Results
}

\author{
Meraj A, MS, M Zahid, D.Orth, MS, S Ahmad, MS \\ Department of Orthopaedics, J.N. Medical College, Aligarh Muslim University, Aligarh, India
}

\begin{abstract}
The operative treatment of displaced intraarticular calcaneal fractures has been a controversial topic. Soft tissue conditions and concomitant disease must be considered in these patients. The minimally invasive sinus tarsi approach is a valid therapeutic solution that guarantees stability, anatomic reduction of the fracture and soft tissue preservation. Twenty-five closed calcaneal fractures in 20 patients were treated with open reduction and internal fixation using the sinus tarsi approach (including fixation with cannulated screws). All fractures healed by the time of final follow-up at 18 months. The time to union judged both clinically and radiographically, averaged 3 months. Mean Maryland foot scores were: 95 in type II; 91 in type III; and 83 in type IV fractures. Advantages offered by this new approach include a less invasive incision while still permitting good visualization and anatomic reduction of articular surfaces and with few complications.
\end{abstract}

Key Words:

Intraarticular calcaneal fractures, sinus tarsi approach, Sanders classification

\section{INTRODUCTION}

Fractures of calcaneum make up a large proportion of major fractures of the tarsal bones and have repeatedly been shown to be associated with significant and prolonged disability. Intraarticular calcaneum fractures of calcaneum comprise $60 \%$ of all tarsal injuries and $75 \%$ of all calcaneal fractures. Displaced intraarticular fractures involving the posterior articular facet, (i.e., the subtalar joint) account for the majority of these fractures. These fractures are uniformly caused by an axial load mechanism such as a fall from height or a motor vehicle accident and may be associated with other axial load injuries, such as lumbar, pelvic and tibial plateau fractures. Numerous controversies surround the management of patients with calcaneal fractures. Clinical evidence supporting operative treatment for selected patient groups is limited, whereas long-term complications and adverse outcomes are frequently documented ${ }^{1}$. The history of treatment of these fractures is characterized by periods of enthusiasm for surgical intervention followed closely by periods of advocacy for closed treatment modalities.
The literature suggests that the outcome after non-operative management has been universally poor. The major cause of poor results result from difficulty in maintaining reduction and loss of position, which can lead to broadening of the hind foot, decreased motion in the subtalar joint, muscle imbalance, impingement of peroneal tendons or secondary osteoarthritis. Historically, there has been much debate over the best approach for treating these fractures ${ }^{2.3}$.

The most common treatment has been open reduction and internal fixation via a lateral extensile approach, but this technique is not without complications. The most frequently used minimally invasive technique for the tongue type fracture was proposed by Westhues in 1935, modified by Gissane, and propagated by Essex-Lopresti ${ }^{4}$. We have used a minimally invasive technique that permits us to treat complex fractures, thereby decreasing complication rates.

Importantly, the primary objectives in such cases are to restore the congruity of the posterior facet and the subtalar joint, to restore the height of the calcaneus (Bohler's angle), to re-establish the integrity of calcaneocuboid joint, to decompress retro-peroneal space and to avoid varus or valgus deformity.

\section{MATERIALS AND METHODS}

This was a prospective study conducted between March 2009 and June 2011, on patients presenting to the outpatient or emergency department of our institution with displaced intraarticular fractures of the calcaneum. Patients of either sex between the ages of 15-60-years with closed, displaced intraarticular fractures of the calcaneum were included in the study. Patients with open fracture of the calcaneum, other fractures of ipsilateral on contralateral limb, and/or other conditions that might affect their gait (paraplegia, quadriplegia, cerebrovascular accidents) were excluded from the study. Following application of inclusion and exclusion criteria 20 patients ( 25 calcaneal fractures) were included in the study. Preoperative assessment included radiographs (lateral, axial and Broden's view) of both ankles and computed tomography (CT) imaging of the affected ankle. Lateral radiographs were used to assess the Bohler's angle and angle of Gissane, loss of height of the calcaneum and 
displacement of the posterior facet (Figure 1a). Axial radiographs were used to assess the increase in width of calcaneum and angulation of tuberosity fragment (Figure 1b). Broden's view was used to evaluate the articular surfaces of the posterior facet of the calcaneum. CT scan of the ankle was used to assess size and number of fracture fragments, displacement of posterior facet and sustentaculum tali and impingement of lateral malleolus on the calcaneal tuberosity (Figure 1c). Fractures were subdivided based on Sander's classification. After preoperative assessment, patients underwent operative procedures within 5 days; in case of excessive swelling, surgery was delayed for 10-14 days in order to allow soft tissue swelling to resolve. In such cases, the foot was placed in a posterior plaster splint and the limb was elevated. Surgery was undertaken after the oedema had settled completely and the wrinkle sign appeared.

\section{Surgical technique}

Surgery was performed with tourniquet with surgical time varying from 50 to $80 \mathrm{~min}$; all patients were treated with cefoperazone for antibiotic prophylaxis. Using the image intensifier and with the patient in lateral position, the fracture was visualized both in lateral and axial view. One Steinmann pin was inserted in the calcaneal tuberosity from the lateral side and then traction toward the bottom was applied to correct the calcaneal valgus. Subsequently, an italic S incision was executed at the sinus tarsi, anteriorly to the lateral malleolus and continued until the calcaneocuboid joint was visible.

The posterior facet was reduced using a curved periosteal elevator, which permits elevation from the calcaneal body. Fixation was obtained using $4 \mathrm{~mm}$ cannulated screws. The reduction was temporarily stabilized with a $\mathrm{K}$ wire and then checked with the intensifier. The anterior facet was reduced and stabilized with $4 \mathrm{~mm}$ cannulated screws, and reduction of the medial wall was obtained with a curved periosteal elevator introduced in the sinus tarsi. The sustentaculum tali was reduced with two $4 \mathrm{~mm}$ cannulated screws positioned from lateral to medial. Lastly, percutaneous fixation of cannulated screws through the posterior tuberosity directed toward the calcaneocuboid joint was performed with screw lengths $60-70 \mathrm{~mm}$ in size.

The wound was irrigated with normal saline and closed in layers. A below knee Plaster of Paris (POP) slab was applied for 2 weeks. Non-weight bearing ambulation was started on the 3 rd postoperative day. Sutures were removed at 2 weeks and a new below knee POP cast was applied for an additional 6 weeks. The cast was then removed and ankle physiotherapy was started. Weight bearing was started at $10-$ 12 weeks postoperatively. All patients were followed at 6 weeks, 12 weeks, 18 weeks, 24 weeks and then at 3-month intervals up to the final visit
Clinical assessment included measurement of the range of motion of the ankle and subtalar joint. Radiological assessment included measurement of Bohler's angle, the angle of Gissane and height of the calcaneum in lateral view of ankle, width of the calcaneum on the axial view and the adequacy of reduction of the posterior articular facet on Broden's view of the ankle (Figure 1d-1e). Subjective assessment involved use of the 100 point Maryland foot scoring system by asking patients about discomfort/pain during activity and rest, ability to stand and walk, ability to return to work, duration of absence from work and any change in the size of the shoe. An overall rating of excellent (90-100), good (80-89), fair (65-79) and poor (below 64) was assigned to each fracture.

\section{RESULTS}

Twenty-five displaced intraarticular calcaneal fractures in 20 patients were included in the study, 15 patients had unilateral fracture whereas 5 patients had bilateral fracture. Fourteen patients were male and 6 were females. In 17 patients (85\%), the mechanism of injury was fall from height, whereas in 3 patients the injury was due to road traffic accident. All fractures were subdivided according to Sander's classification based on CT scan findings (Figure 1c). Six fractures were type 2, eighteen fractures were type 3 and one was type 4 . Average time delay from injury to surgery was 10.5 days (range, 4- 14 days). Mean follow up was 12 months (range 6-18 months). All fractures healed without any major complication. Time to union, judged both clinically and radiologically, averaged 3 months (range, 2-4 months). Surgery succeeded in restoring various parameters such as Bohler's angle, the angle of Gissane, as well as the height and width of the calcaneum to near normal. Preoperatively, the mean Bohler's angle and angle of Gissane were $5.13^{\circ} \quad$ (range, $2^{\circ}-18^{\circ}$ ) and $113.57^{\circ}$ (range, $95^{\circ}-135^{\circ}$ ) respectively, which improved postoperatively to $20.47^{\circ}$ (range, $14^{\circ}-35^{\circ}$ ) and $104.95^{\circ}$ (range, 95 $5^{\circ}-115^{\circ}$ ). The mean preoperative calcaneal height was $39.15 \mathrm{~mm}$, which improved to $41.15 \mathrm{~mm}$ postoperatively; the mean preoperative calcaneal width was $37.46 \mathrm{~mm}$, which improved to $35.42 \mathrm{~mm}$ postoperatively.

There were no cases of infection, hardware failure, wound dehiscence or other soft tissue complications. Patients were evaluated using the Maryland foot score - the mean score was 95 in type 2 fractures, 91 in type 3 fractures and 83 in type 4 fractures.

\section{DISCUSSION}

Surgical treatment is the only viable possibility for comminuted extraarticular or intraarticular fractures. Complication rates vary from $10-20 \%$, and include wound dehiscence (14\% of cases), deep infection (incidence range, 

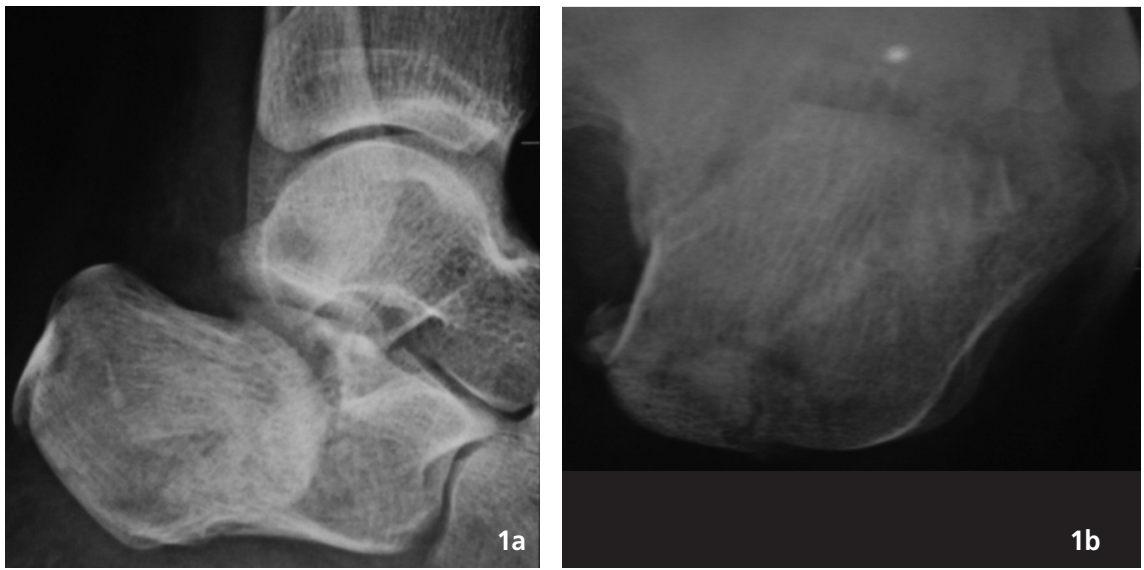

Fig. 1a \& 1b: Preoperative lateral radiographic view of the ankle and axial view of the calcaneum showing displaced intraarticular fracture of the calcaneum with widening.

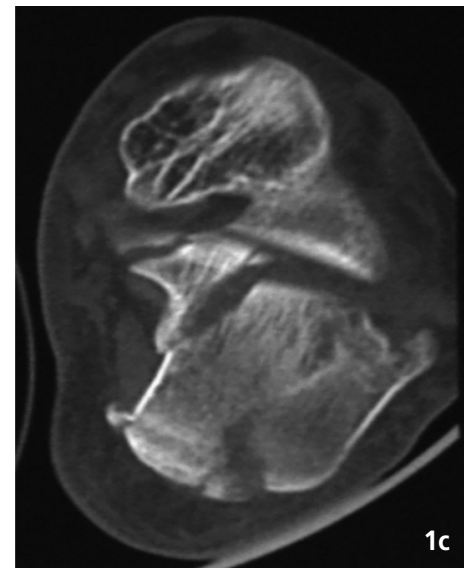

Fig. 1c: Preoperative computed tomography (CT) image of the same ankle showing a displaced intraarticular fracture of the calcaneum with fracture of the sustentaculum tali.
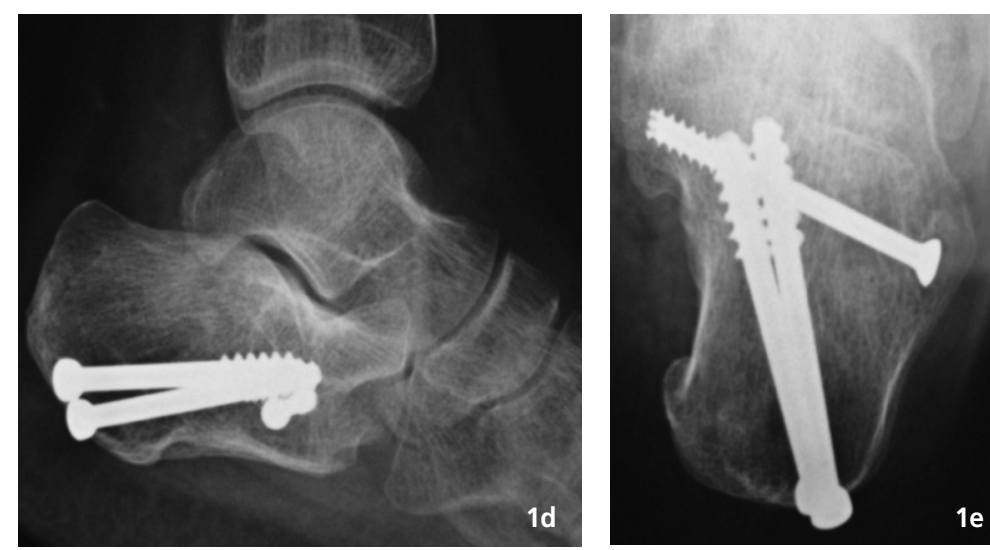

Fig. 1d \& 1e: Twelve weeks postoperative radiographic lateral view of the same ankle and axial view of the calcaneum showing fracture healing with restoration of Bohler's angle, angle of Gissane and calcaneal width.

$1.3-8.5 \%$ ), peroneal tendinitis (incidence, $4 \%$ ), tarsal tunnel syndrome (incidence, $2 \% 0$ or compartmental syndrome (incidence, $2 \%)^{5}$. Once the fracture is classified and the soft tissue condition is evaluated, appropriate treatment must be considered; possibilities include reduction and stabilization with plate and screws or screws alone, primary arthrodesis, or conservative treatment. Surgical results are dependent upon the timing of surgery; according to Sanders, the correct timing is between 7 and 10 days from trauma, because if treated earlier there is risk of compartmental syndrome or soft tissue damage, and if treated later the results could be otherwise unsatisfactory.

Several surgical techniques have been described ${ }^{6,7,8}$. Medial access permits direct reduction of the body of the sustentaculum tali but not of the posterior facet or the anterolateral fragment; lateral access permits allows direct operative access to the posterior facet and the anterolateral fragment and indirectly to the body of the sustentaculum tali.
Combined medial and lateral access enables perfect visualization of the fracture but there is a risk of compromising vascularity; extended lateral access enables avoidance of damage to peroneal tendons and the sural nerve 9 . These treatments enable good results in $50-70 \%$ of the cases, but they are burdened by significant complication rates. Minimally invasive treatment of calcaneal fractures was introduced by Westhues ${ }^{10}$ in 1934 and allows for percutaneous traction with a $\mathrm{K}$ wire introduced in the calcaneal tuberosity and maintenance in a plaster cast.

Mini-open techniques are generally used in patients in critical condition (polytraumatised) and in patients in whom open reduction and internal fixation (ORIF) would be contraindicated, as in elderly patients, smokers, and those with decompensated diabetes, immunodeficiency or soft tissue injuries ${ }^{11,12}$. In such cases, these techniques have been shown to be valid and have satisfactory results. Less invasive fixation has the advantage of correcting the alignment of the 
posterior part of the foot and the articular congruity as well as with classic fixation, but with less damage to soft tissue, thereby decreasing the risks of complications. This fixation is executed with percutaneous cannulated screws, as first described by Essex-Lopresti ${ }^{4,13}$. Other treatments using external fixation with the Ilizarov technique have been described but are reserved for complex fractures or severely compromised patients ${ }^{2}$.

The minimally invasive technique through a sinus tarsi approach permits rebuilding of the articular surface of the anterior and posterior facet, resulting in decreased risk of infection or hardware failure. With a technique similar to ours, Holmes used an incision from the apex of the peroneal malleolus to the base of the fourth metatarsal, thus allowing access to the sinus tarsi, the subtalar joint and visualisation of joint facets. A second mini-incision is executed anteriorly to the calcaneal tuberosity and posteriorly to peroneal tendons on the lateral side. Ebraheim et al. ${ }^{9}$ reported a technique using small incisions at the sinus tarsi to visualise and reduce the fracture of the posterior facet and of the anterolateral fragment. The tuberosity is then reduced with a Steinmann pin at the primary fracture line. These authors suggest that this type of incision is advantageous because it also allows the surgeon to perform subtalar arthrodesis from the same approach if necessary. Similar to the Holmes report, we have never had any infection using the sinus tarsi technique; Ebraheim reports some experience with infection using this technique.
The functional results presented in this study are similar to those reported for open reduction and internal fixation (ORIF) groups in the literature, but appear to be better than those for conservatively treated patients ${ }^{6,7,8,9,13}$. This study confirms that the function of the calcaneus and subtalar joint can be restored by percutaneous reduction and fixation in patients with a displaced intraarticular calcaneal fracture. The good outcome scores and the preservation of the subtalar range of motion at 3-year follow-up with the minimally invasive procedure indicate little benefit of open procedures, over percutaneous reduction and fixation.

\section{CONCLUSION}

Sinus tarsi approach is a less invasive method for fixation of calcaneal fractures. It permits good visualization of the fracture, and allows anatomic reduction of articular surfaces and can also be used to perform subtalar arthrodesis when necessary. This is a valid option of treatment for displaced intra-articular calcaneal fractures in young active adults. 


\section{REFERENCES}

1. Randle JA, Kreder HJ, Stephen D, Williams J, Jaglal S, Hu R. Should calcaneal fractures be treated surgically? A meta-analysis. Clin Orthop. 2000; 377: 217-27.

2. Khaled ME, Mohamed FA. Management of calcaneal fracture using the Ilizarov technique. Clin Orthop. 2005; 439: 215-20.

3. Letournel E. Open treatment of acute calcaneal fractures. Clin Orthop. 1993; 290: 60-7.

4. Essex-Lopresti P. The mechanism, reduction technique, and results in fractures of the os calcis. Br J Surg. 1952; 39: 395-419.

5. Crosby LA, Fitzgibbons TC. Open reduction and internal fixation of type II intra-articular calcaneus fractures. Foot Ankle Int. 1996; 17: 253-8.

6. Benirschke SK, Sangeorzan BJ. Extensive intraarticular fractures of the foot. Surgical management of calcaneal fractures. Clin Orthop. 1993; 292: 128-34.

7. Carr JB. Mechanism and pathoanatomy of the intra-articular calcaneal fractures. Clin Orthop. 1993; 290: 36-40.

8. Harvey EJ, Grujic L, Early JS, Benirschke SK, Sangeorzan B. Morbidity associated with ORIF of intra-articular calcaneus fractures using a lateral approach. Foot Ankle Int. 2001; 22(11): 868-73.

9. Ebraheim NA, Elgafy H, Sabry FF, Freih M, Abou-Chakra IS . Sinus tarsi approach with trans-articular fixation for displaced intra-articular fractures of the calcaneus. Foot Ankle Int. 2000; 21(2): 105-13.

10. Westhues H. Eine neue Behandlungsmethode der Calcaneusfrakturen. Zugleichein Vorschlag zur Behandlung der Talusfrakturen. Zentralbl Chir 1935; 35: 995-1002.

11. Levine DS, Helfet DL. An introduction to the minimally invasive osteosynthesis of intra-articular calcaneal fractures. Injury. 2001; 32 (1): 51-4.

12. Rammelt S, Zwipp H.Calcaneus fractures. Facts, controversies and recent developments. Injury. 2004; 35: 443-61.

13. Tornetta PIII. The Essex-Lopresti reduction for calcaneal fractures revisited. J Orthop Trauma. 1998; 12: 469-73. 